Artículo

\title{
Respuesta de la composta sobre la fertilidad, humedad del suelo, contenido nutrimental en hojas y productividad en olivo
}

\author{
Rubén Macías Duarte ${ }^{1 \S}$ \\ Raúl Leonel Grijalva Contreras ${ }^{1}$ \\ Fabián Robles Contreras ${ }^{1}$ \\ Arturo López Carvajal ${ }^{1}$ \\ Fidel Núñez Ramírez ${ }^{2}$ \\ ${ }^{1}$ Campo Experimental Costa de Hermosillo-Sitio Experimental Caborca-INIFAP. Avenida S núm. 8 norte \\ H. Caborca, Sonora, México. CP. 83600. Tel. 55 38718700, ext. 81105. (macias.ruben@inifap.gob.mx; \\ grijalva.raul@inifap.gob.mx; robles.fabian@inifap.gob.mx; lopez.arturo@inifap.gob.mx). ${ }^{2}$ Instituto de \\ Ciencias Agrícolas-Universidad Autónoma de Baja California. Ejido Nuevo León, Mexicali, Baja \\ California. (fidel.nunez@uabc.edu.mx). \\ ${ }^{\S}$ Autor para correspondencia: macias.ruben@ @inifap.gob.mx.
}

\section{Resumen}

El uso de residuos orgánicos representa una buena alternativa para la nutrición vegetal del olivo. El objetivo fue evaluar el efecto de la aplicación de composta en las condiciones físicas y químicas del suelo, nivel nutrimental en hojas, humedad del suelo y productividad del olivo en regiones desérticas. La evaluación se realizó en una huerta localizada en la región de Caborca, Sonora, México durante los años 2018 y 2019. El cultivar utilizado fue Manzanilla de Sevilla, con un distanciamiento de $10 \times 5 \mathrm{~m}$ (200 árboles ha $\left.^{-1}\right)$. Los tratamientos evaluados fueron: 1) composta + paja de trigo $(\mathrm{C}+\mathrm{P})$; y 2 ) testigo absoluto ( fueron: fertilidad de suelo, contenido nutrimental en hojas, humedad del suelo, rendimiento y calidad del fruto. El diseño experimental usado fue completamente al azar con cuatro repeticiones. Con la aplicación de composta y paja de trigo $(\mathrm{C}+\mathrm{P})$ se incrementó la concentración de la mayoría de los nutrimentos edáficos, el contenido de materia orgánica, la capacidad de intercambio catiónico y la salinidad del suelo. En planta incrementó el contenido nutrimental en hojas. Por otro lado, la humedad de suelo, el rendimiento y calidad de aceitunas no fueron afectados estadísticamente con la incorporación al suelo de composta y paja de trigo $(\mathrm{C}+\mathrm{P})$, con relación al testigo (T). A pesar de una mejora en el suelo la aplicación de composta en dos años no ha incrementado la productividad en el olivo.

Palabras clave: Olea europaea L., calidad, materia orgánica.

Recibido: septiembre de 2020

Aceptado: noviembre de 2020 


\section{Introducción}

La incorporación de residuos orgánicos al suelo, como fuente de nutrición vegetal en la producción de cultivos y a menores costos, representa una excelente alternativa para este fin. El compostaje sirve para convertir estos residuos en un producto de gran beneficio que se incorpora al suelo como abono orgánico, el cual mejora las condiciones físicas y nutrimentales del mismo (Trinidad y Velasco, 2016). El efecto más significativo que se produce sobre un suelo cuando se le adicionan residuos orgánicos, es el aumento de su contenido en materia orgánica.

Al utilizar los abonos orgánicos en forma rutinaria en los suelos agrícolas aumenta el contenido de materia orgánica a mediano y largo plazo y, con ello, la disponibilidad de nutrimentos. Esto es, las

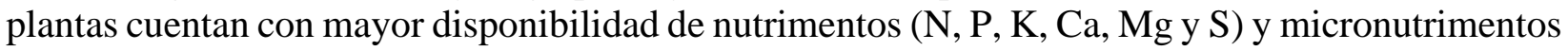
( $\mathrm{Fe}, \mathrm{Mn}, \mathrm{Cu}, \mathrm{Zn} \mathrm{Ni}, \mathrm{B}$, Mo y $\mathrm{Cl}$ ) con una mayor capacidad de intercambio catiónico (CIC) (Trinidad y Velasco, 2016). Huddleston y Ronayne (1990), mencionan que las mejores respuestas de los suelos a la aplicación de abonos orgánicos son aquellos de textura media a gruesa, como los suelos francos, areno-franco, arcillo-franco y arenosos.

La adición de abonos orgánicos al suelo, mejoran sus condiciones físicas y químicas y estimulan la actividad biológica (Bulluck et al., 2002; Roldán et al., 2003; Hargreaves et al., 2008; Lovieno et al., 2009). Los abonos orgánicos ayudan a mejorar la estructura, estabilidad de agregados, retención de humedad del suelo y a disminuir la pérdida de nutrimentos reduciendo la escorrentía del agua de riego y lluvia y así evitar la erosión del suelo (Bastida et al., 2007; Tarango, 2010; Arenas et al., 2015). También aumentan la capacidad del suelo para retener cationes intercambiables (López-Piñeiro et al., 2007; Weber et al., 2007). La velocidad de mineralización de la materia orgánica (MO) depende de la naturaleza física y química de los residuos orgánicos, de los microorganismos que intervinieron en la descomposición y de las condiciones fisicoquímicas del proceso (humedad, aireación, temperatura y pH) (Trinidad y Velasco, 2016).

Dos de los resultados de la mineralización importantes en la MO son los ácidos húmicos y fúlvicos, los cuales son los responsables de muchas de las mejoras que ejerce el humus (Brunetti et al., 2005). Al respecto Brunetti et al. (2005) mencionan que el contenido de ácidos húmicos en el suelo, aumentaron cuando se incorporaron residuos vegetales procedentes de árboles de olivo.

Por otro lado, la adición de MO al suelo favorece la proliferación de los microorganismos benéficos, como son las bacterias, hongos y actinomicetos. Estos microorganismos participan en los diferentes procesos de degradación de la MO, oxidación y reducción de nutrimentos e inhibición de algunos patógenos del suelo Romero-Lima (1997); Ros et al. (2008). Asimismo, representa un potencial para controlar patógenos del suelo (Hoiting et al., 1991; Hadar y Mandelbaun, 1992).

En el cultivo y producción de olivo bajo las condiciones de suelo y manejo en la región de Caborca, Sonora, no se cuenta con la información necesaria y adecuada sobre la capacidad productiva del olivo. La aplicación de abonos orgánicos (composta) como fuente de fertilizante, sin el uso de fertilizantes químicos. El objetivo del presente trabajo fue determinar el efecto de la aplicación de composta en las condiciones físicas, químicas del suelo, nivel nutrimental en las hojas de las plantas, humedad del suelo, y productividad del olivo en regiones desérticas. 


\section{Materiales y métodos}

\section{Descripción del área de estudio}

El trabajo se realizó en terrenos de la Sociedad de Producción Rural (SPR) 'Campo Aguilar' localizado en la región de Caborca, Sonora, ubicado a los $30^{\circ} 48^{\prime} 49^{\prime}$ ' de latitud norte y $112^{\circ} 54^{\prime}$ $18^{\prime \prime}$ de longitud oeste y una altitud de $44 \mathrm{~m}$. El clima es desértico con una temperatura media anual de $22{ }^{\circ} \mathrm{C}$. Enero es el mes más frio con $4.6{ }^{\circ} \mathrm{C}$ y julio el más caliente con $40.2^{\circ} \mathrm{C}$. La precipitación anual es menor a $200 \mathrm{~mm}$ con una evaporación superior a $2300 \mathrm{~mm}$ anuales (Robles, 2001).

\section{Características del suelo}

El suelo del lote comercial de olivo donde se estableció la evaluación presentó una textura franca en la capa superficial $(0-30 \mathrm{~cm})$ y una textura franco-arenosa en la capa más profunda $(30-60 \mathrm{~cm})$. Es bajo en contenido de materia orgánica (0.6\%), buen drenaje, un pH ligeramente alcalino (7.3) y pobre en nitrógeno $(5.8 \mathrm{ppm})$ y fósforo $(10.1 \mathrm{ppm})$.

\section{Manejo agronómico}

La evaluación se realizó en un huerto de olivo de ocho años durante dos ciclos (2018 y 2019). El cultivar utilizado fue Manzanilla de Sevilla, con marco de plantación rectangular de 10 x 5 m (200 árboles $\mathrm{ha}^{-1}$ ). El experimento se estableció bajo el sistema de riego por goteo. Se utilizaron dos mangueras con goteros con $1 \mathrm{~m}$ de separación entre ellos, situadas sobre la superficie del suelo a cada lado de la línea de plantación, con tres goteros por línea con un gasto de $8 \mathrm{~L} \mathrm{~h}^{-1}$ (seis goteros por árbol). El riego se aplicó cada semana con una duración de 12 a 24 h y la lámina aplicada fue de $12000 \mathrm{~m}^{3} \mathrm{ha}^{-1}$.

El resto del manejo agronómico fue realizado por el productor cooperante. Como se trata de una huerta para producir aceituna orgánica solo se aplicaron productos autorizados. El único agroquímico aplicado fue Spinosad $\left(1 \mathrm{~L} \mathrm{ha}^{-1}\right)$ en dos ocasiones por año para el control de la mosca del olivo (Bactrocera oleae). Además, en forma general en toda la huerta, se realizaron 5 aplicaciones por año de lixiviado de té de lombriz de la especie californiana (Eisenia foetida) en dosis de $5 \mathrm{~L} \mathrm{ha}^{-1}$.

Las malezas perenes que se presentaron fueron: Correhuela (Convolvulus arvensis L.), estafiate (Ambrosia confertifolia D C.), chamizo (Atriplex canescens (pursh) Brandegee) y el control de ellas se realizó por medio de podas mecánicas con una cuchilla acoplada a un tractor, las malezas cortadas fueron esparcidas sobre la superficie del suelo.

\section{Tratamientos evaluados}

Los tratamientos evaluados fueron: 1) aplicación de composta más paja de trigo (C+P) y 2) testigo sin aplicación de composta ni paja de trigo (T). En el tratamiento $\mathrm{C}+\mathrm{P}$, se aplicaron $60 \mathrm{~kg}$ de composta más $13 \mathrm{~kg}$ de paja de trigo por árbol en el mes de noviembre de 2017. Posteriormente en junio de 2018 se realizó la segunda aplicación solamente de composta, en dosis de 120 (kg árbol $^{-1}$ ). La aplicación se realizó sobre la superficie del suelo, debido a que en frutales es difícil 
incorporarla en el perfil del suelo (Cortés, 2011). La composta y la paja de trigo se aplicaron sobre una zanja de aproximadamente $20 \mathrm{~cm}$ de profundidad, quedando sin cubrir (no se enterró) sobre la superficie del suelo, a ambos lados de la línea de plantación, (aproximadamente $1.2 \mathrm{~m}$ del tronco de los árboles) exactamente debajo de la manguera de riego.

La composta utilizada fue producida por el productor cooperante quien utilizó para ello, residuos vegetales producto de la poda de los árboles de olivo, enriquecido con estiércol de ganado bovino. Las características nutricionales de la composta utilizada se presentan en el Cuadro 1. En el testigo (T) no se aplicó composta ni paja de trigo.

Cuadro 1. Contenido nutrimental de la composta utilizada en olivo cultivar Manzanilla de Sevilla durante los años 2018 y 2019.

\begin{tabular}{cccccccccccccccc}
\hline $\mathrm{N}$ & $\mathrm{P}$ & $\mathrm{K}$ & $\mathrm{Mg}$ & $\mathrm{Ca}$ & & $\mathrm{Zn}$ & $\mathrm{Fe}$ & $\mathrm{Mn}$ & $\mathrm{Cu}$ & $\mathrm{Na}$ & $\begin{array}{c}\mathrm{CE} \\
\left(\mathrm{dS} \mathrm{m}^{-1}\right)\end{array}$ & $\begin{array}{c}\mathrm{CIC} \\
\left(\text { meq } 100 \mathrm{~g}^{-1}\right)\end{array}$ & $\mathrm{pH}$ \\
\hline 4.80 & 0.31 & 0.31 & 0.04 & 0.71 & & 4 & 75 & 72 & 1 & 893 & 6.1 & 64.7 & 7.04 \\
\hline
\end{tabular}

\section{Parámetros evaluados y análisis estadístico}

Para medir el efecto de los cambios de las características fisicoquímicas del suelo, en cada tratamiento, se realizó un muestreo a dos perfiles del suelo $(0-30$ y 30-60 cm) en el mes de julio en ambos años. Para la obtención de la muestra, se combinaron cuatro submuestras, una en cada repetición obtenidas a lo largo de la línea de plantación debajo de la manguera de riego. En lo que respecta al árbol, se midió el contenido nutrimental de las hojas de los árboles correspondientes a cada tratamiento. Para esto se realizó un muestreo foliar el día 4 de julio del 2018 y el 11 de julio del año 2019.

El muestreo foliar se realizó de acuerdo con la metodología recomendada por Fernández (2001), para lo cual se recolectaron 100 hojas correspondientes a los árboles de cada tratamiento, localizadas en los cuatro puntos cardinales, a una altura promedio de $2 \mathrm{~m}$ correspondientes a plantas sanas, sin daño de plagas. El contenido de humedad del suelo se determinó a dos profundidades (40 y $80 \mathrm{~cm}$ ) durante el periodo comprendido del 22 de febrero de 2018 hasta el 17 de junio de 2019, por medio de los medidores de humedad del suelo de la marca Watermark modelo Irrometer (CE) colocados a $1 \mathrm{~m}$ del tronco del árbol y a $30 \mathrm{~cm}$ del gotero. Para medir la productividad se determinó el rendimiento $\left(\mathrm{kg}_{\text {árbol }}{ }^{-1}\right)$ el cual fue la media de siete árboles cosechados y la calidad del fruto (peso y diámetro de fruto, así como la relación pulpa hueso) se determinó tomando 100 frutos al azar por repetición al momento de la cosecha. La cosecha se realizó en forma manual el 13 de agosto en el año 2018 y el 3 de septiembre en 2019.

El diseño experimental utilizado fue completamente al azar con cuatro repeticiones, tomándose una hilera con 30 árboles $\left(1500 \mathrm{~m}^{2}\right.$ ) como unidad experimental y la útil los siete árboles centrales $\left(350 \mathrm{~m}^{2}\right)$. Solamente las variables de humedad del suelo, rendimiento y calidad de frutos fueron analizadas estadísticamente usando el programa de análisis de varianza de la FAUANL versión 2.7 (Olivares, 2016). La separación de media se realizó de acuerdo a la diferencia mínima significativa (DMS) al 5\%. 


\section{Resultados y discusión}

\section{Análisis de suelo}

El efecto de la aplicación de composta y paja de trigo sobre el contenido de (MO) en el suelo se muestran en el Cuadro 2. Los resultados indicaron que en el testigo el contenido de MO fue muy pobre en las dos profundidades (Castellanos et al., 2000) y más acentuada en la profundidad de 30$60 \mathrm{~cm}$. La aplicación de composta y paja $(\mathrm{C}+\mathrm{P})$, incrementó la $\mathrm{MO}$ en la capa superficial del suelo. Durante los dos años de estudio, el contenido de MO se incrementó de $0.72 \%$ en el testigo, a $2.38 \%$ en el tratamiento $(\mathrm{C}+\mathrm{P})$ en 2018 y de $0.85 \%$ a $2.71 \%$ en 2019 en la profundidad del suelo entre 0 $30 \mathrm{~cm}$. A la profundidad de suelo mayor $(30-60 \mathrm{~cm}$ ) el incremento de MO fue menor (Cuadro 2). Al respecto, Trinidad y Velazco (2016) mencionan que con la aplicación de residuos orgánicos al suelo se aumenta el contenido de MO y con ello la disponibilidad de nutrientes.

La concentración de sales en el suelo (CE), se incrementó, debido a la aplicación del tratamiento $\mathrm{C}+\mathrm{P}$, principalmente en la capa superficial del suelo, debido a que debido a que uno de los componentes de la composta fue el estiércol de bovino y al respecto Seguel et al. (2013) mencionan que la aplicación de estiércol al suelo puede incrementar la salinidad. En el año 2018 en la profundidad de 0-30 cm el contenido de sales en el suelo aumentó de 1.61 a $3.13 \mathrm{dS} \mathrm{m}^{-1}$ con la aplicación del tratamiento $\mathrm{C}+\mathrm{P}$, mientras que en la capa $30-60 \mathrm{~cm}$ el incremento fue menor de 1.74 a $2.2 \mathrm{dS} \mathrm{m}^{-1}$ para ambos tratamientos (Cuadro 2).

En el año 2019, la concentración de sales en el suelo bajó en relación con el año anterior para ambos tratamientos. Esta reducción fue debida probablemente al efecto de lavado de sales por el agua de riego aplicado durante los dos ciclos sobre el tratamiento donde se aplicó el tratamiento $\mathrm{C}+\mathrm{P}$. Al respecto se menciona que el método más utilizado para la recuperación de suelos salinos es el lavado o la lixiviación de las sales solubles con agua de baja salinidad (Aguilar, 2013).

La conductividad eléctrica (CE) refleja el contenido de sales solubles en el agua y en el suelo y cuando su contenido es alto, se presentan efectos fitotóxicos en el crecimiento de las plantas. Sin embargo, Grijalva et al. (2010) mencionan que el olivo es tolerante la salinidad del suelo y del agua sin detrimento en su productividad. Por otro lado, Castellanos et al. (2000), indican que el olivo puede soportar concentraciones de hasta $4 \mathrm{dS} \mathrm{m}^{-1} \sin$ afectar el rendimiento. Por consiguiente, los valores de $\mathrm{CE}$ detectados por el análisis de laboratorio en el presente trabajo no representan un problema para la producción del cultivo bajo estas condiciones.

La capacidad de intercambio de cationes (CIC) fue otro de los parámetros que se incrementó con la aplicación del tratamiento $\mathrm{C}+\mathrm{P}$ principalmente en la capa superficial 0-30 $\mathrm{cm}$ en ambos años de evaluación con relación al testigo en el año 2018 (Cuadro 2). Al respecto Guerrero (1996) menciona que la presencia las sustancias húmicas incrementan la capacidad de intercambio catiónico (CIC) de los suelos al formar complejos arcilla-húmicos (Guerrero, 1996).

La capacidad de intercambio catiónico designa los procesos de: a) adsorción de cationes por el complejo de cambio desde la solución suelo; y b) liberación de cationes desde el complejo de cambio hacia la solución suelo. Esta propiedad es atribuida a la arcilla (coloide mineral) y al humus (coloide orgánico), de manera que la CIC, está influenciada por: la cantidad y tipo de arcilla, la cantidad de humus y el pH o reacción del suelo (Sánchez, 2007). 
Con respecto a los nutrimentos analizados (Cuadro 2) en los dos años de evaluación, la mayor variación de estos se dio en la capa superficial $(0-30 \mathrm{cn})$ con el tratamiento $\mathrm{C}+\mathrm{P}$, debido a que la composta se aplicó sobre la superficie sin incorporarla en el suelo, por lo que los efectos de esta aplicación fueron mínimos en la capa 30-60 cm. En los dos años de evaluación, la aplicación del tratamiento $\mathrm{C}+\mathrm{P}$ incrementó el contenido de los nutrimentos analizados en ambas profundidades.

Sin embargo, el contenido de $\mathrm{N}$ y $\mathrm{P}$ en el suelo, se encuentran muy deficientes en ambas profundidades en el año 2019 (Cuadro 2). Al respecto, Giese et al. (2011) mencionan que, en zonas áridas y semiáridas, como el noroeste de México, la producción orgánica está limitada por la escasez de nitrógeno en el suelo. Por otro lado, Heeb et al. (2005) mencionan que, al aplicar composta al suelo, en el primer año, existe una disponibilidad para la planta de $70-80 \%$ de fosforo y de $80-90 \%$ de potasio contenidos en esta, no así el nitrógeno el cual debe mineralizarse para ser absorbido por las plantas, mineralizándose en el primer año solo el 11\%, por lo cual si no es abastecido apropiadamente se genera una deficiencia de este elemento.

Cuadro 2. Contenido nutrimental del suelo en dos tratamientos a dos profundidades en el cultivar de olivo Manzanilla de Sevilla en la región de Caborca, Sonora durante 2018 y 2019.

\begin{tabular}{|c|c|c|c|c|c|c|c|c|c|}
\hline \multirow{3}{*}{ Parámetro } & \multicolumn{4}{|c|}{ Profundidad 0-30 cm } & \multicolumn{4}{|c|}{ Profundidad 30-60 cm } & \multirow{3}{*}{$\begin{array}{c}\text { Nivel } \\
\text { Referencia }\end{array}$} \\
\hline & \multicolumn{2}{|c|}{ Testigo } & \multicolumn{2}{|c|}{$\mathrm{C}+\mathrm{P}$} & \multicolumn{2}{|c|}{ Testigo } & \multicolumn{2}{|c|}{$\mathrm{C}+\mathrm{P}$} & \\
\hline & 2018 & 2019 & 2018 & 2019 & 2018 & 2019 & 2018 & 2019 & \\
\hline $\mathrm{MO}(\%)$ & 0.72 & 0.85 & 2.38 & 2.71 & 0.09 & 0.16 & 0.27 & 0.29 & $>2$ \\
\hline $\mathrm{CE}\left(\mathrm{dS} \mathrm{m} \mathrm{m}^{-1}\right)$ & 1.61 & 1.4 & 3.13 & 1.52 & 1.74 & 1.37 & 2.2 & 1.47 & $<4$ \\
\hline CIC (meq $100 \mathrm{~g}^{-1}$ ) & 29.9 & 27.7 & 40.8 & 27.3 & 16.5 & 21.4 & 21.5 & 21.3 & $5-50$ \\
\hline $\mathrm{pH}$ & 7.3 & 8.7 & 7.3 & 8.21 & 7.2 & 8.75 & 7 & 8.77 & 7 \\
\hline $\mathrm{N}-\mathrm{NO}_{3}(\mathrm{ppm})$ & 5.4 & 6.7 & 6.7 & 11.7 & 3.5 & 2.76 & 4.1 & 3.68 & 30 \\
\hline $\mathrm{P}(\mathrm{ppm})$ & 9.5 & 20.3 & 9.9 & 27.7 & 10.4 & 21.3 & 9.9 & 21.6 & 30 \\
\hline $\mathrm{K}(\mathrm{ppm})$ & 494 & 291 & 466 & 293 & 256 & 210 & 572 & 219 & 350 \\
\hline $\mathrm{Ca}(\mathrm{ppm})$ & 3547 & 4171 & 5799 & 4194 & 1896 & 3298 & 2483 & 3222 & 2000 \\
\hline $\mathrm{Mg}(\mathrm{ppm})$ & 883 & 550 & 885 & 504 & 498 & 366 & 618 & 373 & 250 \\
\hline $\mathrm{Na}(\mathrm{ppm})$ & 718 & 375 & 781 & 338 & 516 & 312 & 590 & 357 & $<200$ \\
\hline $\mathrm{CaCO}_{3}(\%)$ & 4 & 5.73 & 4 & 5.1 & 4 & 3.6 & 4 & 5.1 & 1 \\
\hline
\end{tabular}

$\mathrm{z}=$ Castellanos et al. (2000).

\section{Análisis foliar}

El efecto de la aplicación de composta y paja de trigo sobre el contenido nutrimental en los árboles de olivo se muestran en el Cuadro 3. Los resultados del análisis foliar indican que, en el año 2018, con la aplicación del tratamiento $\mathrm{C}+\mathrm{P}$, las hojas de las plantas presentaron un incremento en la mayor parte de los nutrimentos analizados como N, P, K, Ca, Mg, $\mathrm{Na} \mathrm{Fe} \mathrm{y} \mathrm{Zn,} \mathrm{con} \mathrm{excepción} \mathrm{en}$ el contenido de S, Mn y Cu los cuales disminuyeron. En el año 2019 el contenido de nutrimentos en la hoja no presentó diferencias con respecto al año anterior. Raviv et al. (2004-2005), mencionan que la composta contiene considerables cantidades de nutrimentos que pueden suplementar la nutrición de plantas, esto depende del tipo de composta ya que a su vez dependen de las diferentes materias primas utilizadas, por lo que no se puede tomar como una regla. 
Los resultados de los niveles nutricionales obtenidos en el análisis foliar en laboratorio en ambos tratamientos ( $\mathrm{T}$ y $\mathrm{C}+\mathrm{P}$ ) en los dos años de evaluación (Cuadro 3), indican que los árboles no presentaron deficiencias nutricionales en el contenido de $\mathrm{N}, \mathrm{K}, \mathrm{Ca}, \mathrm{Mn}, \mathrm{S}$, fe, Zn, Mn y B (Fernández, 2001). El bajo nivel de P en el suelo se reflejó en el contenido de la hoja el cual presentó un nivel deficiente al igual que el contenido de $\mathrm{Mn}$ y $\mathrm{Cu}$ (Cuadro 3). Por otra parte, regularmente el contenido de nutrimentos en el suelo no siempre está relacionado con el análisis foliar de la planta, ya que se han observado deficiencias de potasio en plantas establecidas en suelos ricos en el mismo elemento cuando existe falta de agua en periodos prolongados de estrés (Rallo, 2001).

Cuadro 3. Contenido nutrimental de hojas de olivo en dos tratamientos en el cultivar de olivo Manzanilla de Sevilla durante los años 2018 y 2019.

\begin{tabular}{ccccccc}
\hline \multirow{2}{*}{ Nutrimento } & \multicolumn{2}{c}{ Testigo $(\mathrm{T})$} & & \multicolumn{2}{c}{ C+P } & Nivel de referencia \\
\cline { 2 - 3 } \cline { 5 - 6 } & 2018 & 2019 & & 2018 & 2019 & \\
\hline $\mathrm{N}(\%)$ & 1.9 & 1.8 & & 2 & 1.78 & $1.5-2$ \\
$\mathrm{P}(\%)$ & 0.09 & 0.1 & & 0.1 & 0.11 & $0.15-0.25$ \\
$\mathrm{~K}(\%)$ & 1.07 & 1.29 & & 1.45 & 1.23 & $1-1.2$ \\
$\mathrm{Ca}(\%)$ & 1.8 & 1.99 & & 2.03 & 1.69 & $1.5-2.5$ \\
$\mathrm{Mg}(\%)$ & 0.2 & 0.15 & & 0.22 & 0.15 & $0.15-0.2$ \\
$\mathrm{~S}(\%)$ & 0.19 & 0.21 & & 0.17 & 0.19 & $0.1-0.2$ \\
$\mathrm{Na}(\%)$ & 0.19 & 0.06 & & 0.24 & 0.08 & $<0.2$ \\
$\mathrm{Fe}(\mathrm{ppm})$ & 121 & 134 & & 190 & 104 & $80-130$ \\
$\mathrm{Zn}(\mathrm{ppm})$ & 24 & 22.5 & & 29 & 19.4 & $15-30$ \\
$\mathrm{Mn}(\mathrm{ppm})$ & 40 & 60.8 & & 36 & 48.5 & $50-130$ \\
$\mathrm{Cu}(\mathrm{ppm})$ & 8 & 7.09 & & 7 & 8.4 & $8-40$ \\
$\mathrm{~B}(\mathrm{ppm})$ & 34 & 42.8 & & 34 & 47.4 & $20-70$ \\
\hline
\end{tabular}

Fecha de muestreo: 04 de julio 2018 y 11 de julio $2019 ;{ }^{\text {z }}=$ Fernández (2001).

\section{Humedad del suelo}

El contenido de humedad del suelo durante el periodo de evaluación se muestra en el Cuadro 4, donde se observó que no hubo diferencias estadísticas significativas entre los tratamientos $\mathrm{T}$ y $\mathrm{C}+\mathrm{P}$. Sin embargo, si hubo diferencias estadísticas entre las dos profundidades del suelo (Cuadro 4). Aún y cuando no hubo diferencias entre tratamientos. El uso de enmiendas como el estiércol o composta favorecen el proceso de infiltración y aumentan la retención de agua y estabilidad de agregados (Trueba, 1996; Seguel et al., 2003).

Los factores que influyeron en el resultado obtenido son, primeramente, el manejo eficiente del agua de riego que realiza el productor cooperante, evitando deficiencias de humedad del suelo durante el periodo de evaluación, por otro lado, la presencia de maleza viva o cortada y esparcida sobre la superficie del suelo en ambos tratamientos, lo cual cubrió y protegió al mismo, evitando que los rayos solares evaporizaran la humedad. Al respecto, Shaxson y Barber (2005) mencionan que las coberturas vegetales, disminuyen la evaporación de agua debido a una mejora en la estructuración superficial, que actúa como colchón antievaporante, además, de tener uso como cobertura del suelo, y poder usarse como fertilizante orgánico (Arancon et al., 2000; Albiach et al., 2001; Madejon et al., 2001). 
El olivo es una especie altamente tolerante a la sequía con relación a otras especies frutales, aunque el uso de riego permite obtener mayores rendimientos (Searles et al., 2011). Esto se debe a que los olivos tienen hojas diseñadas para disminuir su transpiración, ya que cuentan con pelos aparasolados, también llamadas escamas peltadas, que se encuentran tanto en el haz como en el envés de las hojas, aunque en este último en mayor proporción y cubren las estomas (Barranco et al., 2008).

Cuadro 4. Contenido de humedad del suelo a dos tratamientos y dos profundidades (kPa) en olivo en el cultivar Manzanilla de Sevilla durante los años 2018 y 2019.

\begin{tabular}{ccc}
\hline Tratamiento & Profundidad de suelo $(\mathrm{cm})$ & Humedad del suelo $(\mathrm{kPa})$ \\
\hline $\mathrm{T}$ & 40 & $17.1 \mathrm{a}^{\mathrm{z}}$ \\
$\mathrm{C}+\mathrm{P}$ & 80 & $16.8 \mathrm{a}$ \\
$\mathrm{T}$ & 40 & $15.4 \mathrm{~b}$ \\
$\mathrm{C}+\mathrm{P}$ & 80 & $14.1 \mathrm{~b}$ \\
\hline
\end{tabular}

${ }^{\mathrm{z}}=$ medias con la misma letra son estadísticamente iguales (DMS 5\%).

\section{Rendimiento}

Los rendimientos en ambos tratamientos se presentan en el Cuadro 5. La aplicación del tratamiento $\mathrm{C}+\mathrm{P}$ no afectó estadísticamente el rendimiento de los frutos de olivo y fue estadísticamente igual al testigo en ambos años de evaluación. En el año 2019 los rendimientos obtenidos por ambos tratamientos superaron en más de $100 \%$ a los rendimientos obtenidos en el 2018, pero sin diferencias estadísticas. Estos resultados, fueron debido primeramente al fenómeno de la alternancia (Lavee y Avidan, 1994; Rallo et al., 1994) que se presenta en la producción del olivo y en segundo término, al mayor tamaño de planta del huerto en 2019 con respecto al año anterior, lo cual conlleva a una mayor copa vegetativa del árbol y por consiguiente mayor capacidad de producción.

Cuadro 5. Rendimiento de aceituna en la variedad Manzanilla de Sevilla durante 2018 y 2019.

\begin{tabular}{cccccc}
\hline \multirow{2}{*}{ Tratamiento } & \multicolumn{2}{c}{$\left(\mathrm{kg} \mathrm{árbol}^{-1}\right)$} & & \multicolumn{2}{c}{$\left(\mathrm{t} \mathrm{ha}^{-1}\right)$} \\
\cline { 2 - 3 } \cline { 5 - 6 } & 2018 & 2019 & & 2018 & 2019 \\
\hline $\mathrm{T}$ & $41.7 \mathrm{a}^{\mathrm{z}}$ & $108 \mathrm{a}$ & & 8.3 & 21.6 \\
$\mathrm{C}+\mathrm{P}$ & $49.4 \mathrm{a}$ & $118 \mathrm{a}$ & & 9.9 & 23.7 \\
\hline
\end{tabular}

${ }^{\mathrm{z}}=$ medias con la misma letra son estadísticamente iguales (DMS 5\%).

\section{Calidad}

Los parámetros de calidad del fruto (peso, diámetro, longitud de fruto y la relación pulpa-hueso) (Cuadro 6) no presentaron diferencias estadísticas entre tratamientos en los dos años de evaluación. La aplicación del tratamiento $\mathrm{C}+\mathrm{P}$, no afecto la calidad del fruto con respecto al testigo. En el año 2018 el valor de todos los parámetros de calidad fue mayor que en el 2019 y por consiguiente se presentó un marcado incremento en el número de frutos por planta, lo cual ocasionó una mayor competencia por los asimilados producidos por la planta y en consecuencia una reducción en el tamaño de los frutos. 
Investigaciones realizadas por Regni et al. (2016) reportaron que aplicaciones de composta (130 $\mathrm{kg}$ árbol $^{-1}$ ) procedentes de residuos de poda y de residuos de molienda en la extracción de aceite de oliva incrementaron el rendimiento de fruto, aunque sin diferencia significativa y no afectaron las características del fruto.

Cuadro 6. Peso de fruto, diámetro de fruto, longitud de fruto y relación pulpa hueso en aceituna del cultivar de olivo Manzanilla de Sevilla en 2018 y 2019.

\begin{tabular}{|c|c|c|c|c|c|c|c|c|}
\hline \multirow[t]{2}{*}{ Tratamiento } & \multicolumn{2}{|c|}{$\begin{array}{l}\text { Peso de fruto } \\
(\mathrm{g})\end{array}$} & \multicolumn{2}{|c|}{$\begin{array}{l}\text { Diámetro de fruto } \\
(\mathrm{cm})\end{array}$} & \multicolumn{2}{|c|}{$\begin{array}{l}\text { Longitud de fruto } \\
\qquad(\mathrm{cm})\end{array}$} & \multicolumn{2}{|c|}{$\begin{array}{c}\text { Relación } \\
\text { pulpa/hueso }\end{array}$} \\
\hline & 2018 & 2019 & 2018 & 2019 & 2018 & 2019 & 2018 & 2019 \\
\hline $\mathrm{T}$ & $3.8 \mathrm{a}^{\mathrm{z}}$ & $3.5 \mathrm{a}^{\mathrm{z}}$ & $1.7 \mathrm{a}^{\mathrm{z}}$ & $1.4 \mathrm{a}^{\mathrm{z}}$ & $2.2 \mathrm{a}^{\mathrm{z}}$ & $1.8 \mathrm{a}^{\mathrm{z}}$ & $3.9 \mathrm{a}^{\mathrm{z}}$ & $3.8 \mathrm{a}^{\mathrm{z}}$ \\
\hline $\mathrm{C}+\mathrm{P}$ & $3.7 \mathrm{a}$ & $3.5 \mathrm{a}$ & $1.7 \mathrm{a}$ & $1.4 \mathrm{a}$ & $2.2 \mathrm{a}$ & $1.8 \mathrm{a}$ & $3.8 \mathrm{a}$ & $3.8 \mathrm{a}$ \\
\hline
\end{tabular}

${ }^{z}=$ Medias con la misma letra son estadísticamente iguales (DMS 5\%).

Al respecto Burge et al. (1987); Costa et al. (1995) mencionan que el aumento en la carga de fruta está acompañado por una disminución del peso promedio del fruto. Por otro lado, la carga de fruta, en árboles expuestos a las mismas condiciones ambientales, es el principal factor que modula el crecimiento y por lo tanto, el tamaño final de la fruta (Lavee y Wodner, 2004).

\section{Conclusiones}

La aplicación de composta y paja de trigo, al menos durante dos años mejoró las propiedades físicas y químicas del suelo con excepción del contenido de la salinidad e incrementó ligeramente el contenido nutrimental foliar en los arboles de olivo, pero no así el rendimiento y calidad del fruto con respecto al testigo y tampoco el contenido de humedad en el suelo.

\section{Literatura citada}

Aguilar, B. 2013. El suelo de cultivo y las condiciones climáticas. IC Editorial. Málaga, España. $59 \mathrm{p}$.

Albiach, R.; Canet, A.; Pomares, F. and Ingelmo, F. 2001. Organic matter components, agregate stability and biological activity in a horticultural soil fertilized with different rates of two sewage sludges for ten years. Biores. Technol. 77(2):109-114.

Arancon, N. Q.; Edwards, C. A.; Lee, S. S. and Yardim, E. 2000. Management of plant parasitic nematode populations by use of vermicomposts. Ohio State University. 47(17):741-744.

Arenas, A. F. J.; Hervalejo, G. A. y De Luna, A. E. 2015. Guía de cubiertas vegetales en cítricos. Folleto s/n. Sevilla 2015. (Ed.). Instituto de Investigación y Formación Agraria y Pesquera. Consejería de Agricultura, Pesca y Desarrollo Rural. Junta de Andalucía. 12 p.

Barranco, D.; Fernández-Escobar, R. y Rallo, L. 2008. El cultivo del olivo. Madrid, España. Ediciones Mundi-Prensa Coedición Junta de Andalucía. 846 p.

Bastida, F.; Moreno, J. L.; Hernández, T and García, C. 2007. Microbial activity in non-agricultural degraded soil exposed to semiarid climate. Sci. Total Environ. 378(1-2):183-186.

Brunetti, G.; Plaza, C. and Senesi, N. 2005. Olive pomace amendment in mediterranean conditions: effect on soil and humic acid properties and wheat (Triticum turgidum L.) yield. J. Agric. Food Chem. 53(17):6730-6737. 
Bulluck, L.; Brosiud, M.; Evanylo, G. and Ristaino, J. 2002. Organic and synthetic fertility amendments influence soil microbial, physical and chemical properties on organic and conventional farms. Appl. Soil Ecol. 19(2):147-160.

Burge, G. K.; Spence, C. B. and Marshall, R. R. 1987. Kiwifruit: effects of thinning on fruit size, vegetative growth, and return bloom. N. Zealand Journ. Exp. Agric. 15(3):317-324.

Castellanos, J. Z.; Uvalle, B. J. X. y Aguilar, S. A. 2000. Manual de interpretación de análisis de suelos y aguas. Colección INCAPA. Segunda Edición. 226 p.

Cortés, A. 2011. Estabilización física de camellones con palto vía enmiendas orgánicas/cultivos de cobertura, IV Región de Chile. Memoria Ingeniero Agrónomo. Santiago, Chile: Facultad de Ciencias Agronómicas, Universidad de Chile. 45 p.

Costa, G.; Succi, F.; Morigi, M.; Biasi, R. and Galliano, A. 1995. Effetti della carica de gemme e del diradamento dei frutti su quantitá e qualitá della fruttificazione di Hayward (Actinidia deliciosa). Riv. di Frutticolt. 57(4):59-62.

Fernández, E. R. 2001. Fertilización. In: El cultivo del olivo. Barranco, D.; Fernández, E. R. y Rallo, L. (Eds.) Edición Mundi-Prensa, Madrid España. Cuarta Edición. 155-284 pp.

Giese, M.; Gao, Y. Z.; Lin, S. and Brueck, H. 2011 Nitrogen availability in a grazed semi-arid grassland is dominated by seasonal rainfall. Plant Soil. 340(1):157-167.

Grijalva, C. R. L.; Navarro, A. J. A. C. y Fimbres, F. A. 2010. El cultivo del olivo bajo condiciones desérticas del noroeste de Sonora. SAGARPA-INIFAP-CECH-CECAB. Folleto técnico núm. 41.100 p.

Guerrero, A. 1996. El suelo, los abonos y la fertilización de los cultivos. Ediciones Mundi-Prensa, Bilbao, España. 206 p.

Hadar, Y. and Mandelbaum, R. 1992. Suppressive compost for biocontrol of soilborne plant pathogens. Phytoparasitica. 20(S):113-116.

Hargreaves, J.; Add, S.; Warman, P. and Ruparsinghe, H. 2008. The effects of organic amendments on mineral element uptake and fruit quality of raspberries. Plant Soil. 308(1):213-226.

Heeb, A.; Lundegårdh, B.; Ericsson, T. and Savage, G. P. 2005. Effects of nitrate-ammonium and organic nitrogen-based fertilizers on growth and yield of tomatoes. J. Plant Nutr. Soil Sci. 168(1):123-129.

Hoiting, H. A. J.; Inbar, Y. and Boehn, M. J. 1991. Status of compost amended-potting mixes naturally suppressive to soilborne diseases of floricultural. Crop Plant Dis. 75(9):869-873.

Huddleston, J. H. and Ronayne, M. P. 1990. Guide to soil suitability and site selection for beneficial use of sewage sludge. Manual 8. Oregon State University Extension Service. 76 p.

Lavee, S. and Avidan, N. 1994. Protein content and composition of leaves and shoot bark in relation to alternate bearing of olive trees (Olea europaea L.). Acta Hortic. 356(30):143-147.

Lavee, S. and Wonder, M. 2004. The effect of yield, harvest time and fruit size on the oil content in fruits of irrigated olive trees (Olea europaea L.), cvs. Barnea and Manzanillo. Sci. Hortic. 99(3-4):267-277.

López-Piñeiro, A.; Murillo, S.; Barreto, C.; Muñoz, A.; Rato, J. M.; Albarrán, A. and García, A. 2007. Changes in organic matter and residual effect of amendment with two-phase olivemill waste on degraded agricultural soils. Sci.e of the Total Environment. 378(1-2):84-89.

Lovieno, P.; Morra, L.; Leone, A.; Pagano, L. and Afani, A. 2009. Effect of organic and mineral fertilizers on soil respiration an enzyme activity of two Mediterranean horticultural soils. Biology and Fertility of Soils. 45(5):55-561.

Madejon, E. R.; López, J. M.; Murillo, F. and Cabrera, A. 2001. Agricultural use of three (sugarbeet) vinasse composts: Effect on crops and chemical properties of a cambisol soil in the Guadalquivir River Valley (SW Spain). Agric. Ecosyst. Environm. 84(1):55-65. 
Olivares, S. E. 2016. Paquete de diseños experimentales FAUANL. Versión 2.7. Facultad de Agronomía UANL. Marín, NL., México.

Rallo, L. 2001. Fructificación y producción. In: Barranco, D.; Fernández, R. y Rallo, L. (Eds.). El cultivo del olivo. Coedición Mundi-Prensa y Junta de Andalucía. $4^{\text {ta. }}$ Edición. Madrid, España. 261-280 pp.

Rallo, L.; Torreño, P.; Vargas, A. and Alvarado, J. 1994. Dormancy and alternate bearing in olive. Acta Hortic. 356(28):127-136.

Raviv, M. O.; Katan, J.; Hadar, Y.; Yogev, A.; Medina, S.; Krasnovsky, S. and Ziadna, H. 2005. High- nitrogen compost as a medium for organic container grow crops. Bioresour Technol. 96(4):419-42.

Raviv, M.; Medina, S.; Krasnovsky, A. and Ziadna, H. 2004. Organic matter and nitrogen conservation in manure compost of organic agriculture. Compost Sci. Util. 12(1):6-10.

Regni, L.; Nasini, L.; Ilarioni, L.; Brunori, A.; Massaccesi, L.; Agnelli, A. and Proietti, P. 2016. Long term amendment with fresh and composted solid olive mil waste on olive grove affects carbon sequestration by pruning, fuits, and soil. Front. Plant Sci. 7(2420):1-9.

Robles, C. F. 2001. Análisis climatológico de la región de Caborca, Sonora. Ciclo P-V 2001/2001. Reporte técnico (Inédito), INIFAP-CIRNO-CECAB. Caborca, Sonora, México. 7 p.

Roldán, A.; Caravaca, F.; Hernández, T.; Sánchez-Brito, A.; Velázquez, C. and Tiscareño. M. 2003. No-tillage, crop residue additions, and legume cover cropping effects on soil quality characteristics under maize in Patzcuaro Watershed (Mexico). Soil Tillage Res. 72(1):65-73.

Romero-Lima, M. R. 1997. Abonos orgánicos y químicos en producción, sanidad, absorción nutrimental de papa y efecto en el suelo. Tesis de Maestría en Ciencias. Especialidad en Edafología. IRENAT. Colegio de Postgraduados. Montecillo, Estado de México. 139 p.

Ros, M.; Goberna, M.; Pascual, J. A.; Klammer, S. and Insam, H. 2008. 16S rDNA analysis reveals low microbial diversity in community level physiological profile assays. J. Microbiol. Methods. 72(3):221-226.

Sánchez, V. J. 2007. Fertilidad del suelo y nutrición mineral de plantas conceptos básicos-fertitec SA. JSV-GG-FT. Folleto Informativo FERTITEC SA. 19 p.

Searles, S.; Agüero, M. y Rousseaux. M. 2011. El consumo del agua por el cultivo de olivo (Olea europaea L.) en el noroeste de Argentina: una comparación con la Cuenca Mediterránea. Ecología Austral. 21(1):15-28.

Seguel, O. V.; García de Cortázar, A. y Casanova, M. 2003. Variación en el tiempo de las propiedades físicas de un suelo con adición de enmiendas orgánicas. Agricultura Técnica. 63(3):287- 297.

Seguel, O.V.; Baginsky, C. A.; Contreras, J. I. Covarrubias, C.; González, V. and Poblete, L. 2013. Physical properties of a fine textured haplocambid after three years of organic matter amendments management. J. Soil Sci. Plant Nutr. 13(3):690-705.

Shaxson, F. y Barber, R. 2005. Optimización de la humedad del suelo para la producción vegetal: el significado de la porosidad del suelo. Food and Agriculture (FAO). 128 p.

Tarango, R. S. H. 2010. Manejo de la cubierta vegetal en nogaleras con fertirriego. INIFAPCIRNOC- C.E. Delicias. Cd. Delicias, Chihuahua. Folleto técnico núm. 34.24 p.

Trinidad, S. A. y Velasco, V. J. 2016. Importancia de la materia orgánica en el suelo. Agro Productividad. 9(8):52-58.

Trueba, C. S. 1996. Fertilizantes orgánicos y compostas. In: Memorias Agricultura Orgánica: una opción sustentable para el agro mexicano. UACH. Texcoco, México. 163 p. 
Weber, J.; Karczewska, A.; Drozd, J.; Licznar, M.; Licznar, S.; Jamroz, E. and Kocowicz, A. 2007. Agricultural and ecological aspects of a sandy soil as affected by the application of municipal solid waste compost. Soil Biol. Biochem. 39(6):1294-1302. 